Instituto Internacional de Investigación y Desarrollo Tecnológico Educativo INDTEC, C.A.

DOI: https://doi.org/10.29394/Scientific.issn.2542-2987.2020.5.16.16.305-318

OAI-PMH: http://www.indteca.com/ojs/index.php/Revista Scientific/oai

Ensayo Original / Original Essay

\title{
Propuesta pedagógica: JClic como herramienta didáctica en la Educación Primaria
}

Autora: Jerardin del Carmen Ríos Anciani Universidad Nacional Experimental "Rafael María Baralt", UNERMB jerardinrios@gmail.com Zulia, Venezuela https://orcid.org/0000-0003-4926-6435

\section{Resumen}

El uso de las Tecnologías de Información y Comunicación (TIC) en la educación ha sido un pilar fundamental en las políticas educativas del estado venezolano, y llevarlas al aula no ha sido nada fácil, no obstante, pese a la resistencia de algunos docentes tradicionalistas, la realidad demanda docentes que estén a la vanguardia del desarrollo y avance tecnológico, mediante la utilización de herramientas que rompan con la rutina y transformen el proceso educativo. Por ello, se pretende develar el potencial del programa JClic como una herramienta interesante, con facilidad de uso y acceso, la cual la convierte en una herramienta de gran utilidad para los maestros, en la elaboración de actividades multimedia poca inversión de tiempo y de manera sencilla, favoreciendo procesos de enseñanza dinámicos, interactivos, atractivos y significativos, haciendo énfasis en la importancia de la actualización docente para poner en práctica el software JClic, siendo esta una herramienta que complementa su labor en el aula y facilita el aprendizaje de los estudiantes en educación primaria.

Palabras clave: software; tecnología; educación.

Cómo citar este ensayo:

Ríos, J. (2020). Propuesta pedagógica: JClic como herramienta didáctica en la Educación Primaria. Revista Scientific, 5(16), 305-318, e-ISSN: 2542-2987. Recuperado de: https://doi.org/10.29394/Scientific.issn.2542-2987.2020.5.16.16.305-318

Fecha de Recepción: 25-10-2019
Fecha de Aceptación:

20-01-2020
Fecha de Publicación: 05-05-2020 
Instituto Internacional de Investigación y Desarrollo Tecnológico Educativo INDTEC, C.A.

DOI: https://doi.org/10.29394/Scientific.issn.2542-2987.2020.5.16.16.305-318

OAI-PMH: http://www.indteca.com/ojs/index.php/Revista_Scientific/oai

Ensayo Original / Original Essay

\title{
Pedagogical proposal: JClic as a didactic tool in Primary Education
}

\begin{abstract}
The use of Information and Communication Technologies (ICT) in education has been a fundamental pillar in the educational policies of the Venezuelan state, and taking them to the classroom has not been easy, however, despite the resistance of some traditional teachers, reality demands teachers who are at the forefront of technological development and advancement, through the use of tools that break the routine and transform the educational process. Therefore, it is intended to unveil the potential of the JClic program as an interesting tool, with ease of use and access, which makes it a very useful tool for teachers, in the development of multimedia activities, little time and way investment simple, favoring dynamic, interactive, attractive and meaningful teaching processes, emphasizing the importance of teacher updating to implement JClic software, this being a tool that complements their work in the classroom and facilitates student learning in primary education.
\end{abstract}

Keywords: software; technology; education.

How to cite this essay:

Ríos, J. (2020). Pedagogical proposal: JClic as a didactic tool in Primary Education. Revista Scientific, 5(16), 305-318, e-ISSN: 2542-2987. Recovered from:
Date Received:

25-10-2019
Date Acceptance:

20-01-2020
Date Publication:

05-05-2020 


\section{Introducción}

La sociedad actual se caracteriza por estar en un permanente estado de cambios, donde la masificación y avance imparable de las Tecnologías de Información y Comunicación (TIC) han jugado un papel sumamente importante, suscitando una serie de profundas transformaciones en todos los diferentes aspectos en los que se desenvuelve el ser humano. En el ámbito educativo, la forma de enseñar y de aprender ha cambiado, demandando una adaptación urgente en la postura asumida por los profesionales docentes a los nuevos requerimientos que exige la sociedad, por ende, la inserción de herramientas tecnológicas, internet, y software educativos, esto bien, necesita de una readaptación no solo de los contenidos sino de un cambio urgente en las metodologías y estrategias para desarrollar procesos formativos innovadores, integrales y de altísima calidad.

La escuela, se concibe como un espacio crítico para la formación de individuos que de acuerdo a los fines de la educación, expresados en la Ley Orgánica de Educación (LOE), deben ser capaces de integrar conocimientos de diversa índole, científica, humanista y por supuesto tecnológica, en consecuencia se asume, que este espacio debe ser activo, creativo-recreativo y flexible, con el fin de posibilitar a los estudiantes la construcción de aprendizajes significativos mediante el uso de herramientas tecnológicas que coadyuven a alcanzar dichos propósitos de la manera más idónea.

En este marco de ideas, Cacheiro (2011), en relación a la utilización de recursos basados en la tecnología, plantea:

Los recursos TIC para el aprendizaje posibilitan el llevar a cabo los procesos de adquisición de conocimientos, procedimientos y actitudes previstas en la planificación formativa. Tanto los medios didácticos tradicionales como los recursos TIC permiten ofrecer distintas formas de trabajar los contenidos y actividades (pág. 75).

No obstante, aún se observa en los salones de clases, maestros que 
asumen posturas rígidas y mecanizadas del aprendizaje, asimismo, muestran resistencia ante el uso de la tecnología, pareciera entonces, que no han asimilado $\mathrm{ni}$ internalizado las transformaciones sociales y curriculares existentes y prefieren seguir usando los mismos recursos basados en materiales impresos, y la pizarra, dejando fuera del juego el uso de las TIC. Aunado a esto, hay quienes consideran que la inclusión de herramientas Tecnológicas en clase sumaria mayores esfuerzos para su implementación e implicarían dedicar periodos de tiempo que muchos no quieren ceder o no pueden acceder.

A este respecto advierten, Santamarina y Fuentes (2017a): que "Ios docentes son modelos de variedad y corrección expresiva y no pueden quedarse estancados en las prácticas con las que iniciaron su ejercicio profesional" (pág. 295). Actualmente, el paradigma educativo centrado en el docente como transmisor de conocimientos, carece de sentido, de igual manera el uso de estrategias y recursos tradicionales es poco motivador. Si bien es cierto que dichos métodos dieron fruto en su "época", ni la escuela, ni los maestros pueden seguir viviendo en el pasado.

Es importante destacar el hecho que los estudiantes desde temprana edad se familiarizan con todo tipo de aparatos y dispositivos electrónicos de punta, bien sea Computadores, Laptops, iPods, Tablets y Teléfonos inteligentes, razón por la cual, debe existir entonces una educación coherente con las necesidades, intereses, motivaciones y realidad tecnológica en la que están sumergidos los estudiantes que desde la infancia, son usuarios de la tecnología y en ocasiones superan en habilidades para su manejo a sus propios maestros, a lo cual debe sacársele el mayor provecho, puedo agregar. No obstante, ante este panorama también podría afirmar que existen maestros interesados en renovar conocimientos y mejorar su praxis pedagógica con metodologías novedosas que generen resultados exitosos en el proceso de enseñanza-aprendizaje. 
En este contexto, destacan Viñals y Cuenca (2016a): "son muchos los docentes que, por iniciativa propia, han decidido renovarse con el objetivo de seguir preparando al alumnado para el mundo que les toca" (pág. 109); evidentemente sugieren un cambio de actitud, con el fin de actualizar conocimientos y superar sus propios límites. En este sentido, se propone el uso del software JClic como una herramienta que aportaría a las clases de educación primaria el dinamismo, motivación e innovación que tanto necesita, y al docente le proporcionaría todas las facilidades para crear sus propios materiales de trabajo con poca inversión de tiempo, de manera fácil y sencilla.

Tal como plantea, Núñez (2018): este software didáctico, promueve una transformación positiva, por ser un recurso didáctico atractivo para los estudiantes y que necesariamente es de gran provecho para los docentes, en la búsqueda de metodologías efectivas para incentivar y promover aprendizajes dinámicos, salir de lo cotidiano y cambiar la forma tradicional de educar en clase (pág. 87); de allí la importancia que los docentes conozcan las bondades que ofrece el manejo del programa JClic, lo que pueden hacer con él, y atreverse a hacer algo nuevo en pro de una mejor educación. Indudablemente debe existir una integración entre lo teórico y práctico, en combinación con una buena disposición que garantice la apropiación de los conocimientos necesarios para su implementación efectiva en clase.

\section{Desarrollo}

\subsection{Software educativo JClic}

JClic, es un software que permite la creación, y evaluación de actividades educativas multimedia, está desarrollado en la plataforma Java, es gratuito, con variedad de entornos y funciona en diversos sistemas operativos. Esta herramienta permite que los docentes desarrollen múltiples aprendizajes y favorezcan todos los estilos, debido a que permiten que el estudiante las utilice y sea activo con ellas. 
Instituto Internacional de Investigación y Desarrollo Tecnológico Educativo INDTEC, C.A.

DOI: https://doi.org/10.29394/Scientific.issn.2542-2987.2020.5.16.16.305-318

OAI-PMH: http://www.indteca.com/ojs/index.php/Revista_Scientific/oai

Ensayo Original / Original Essay

En relación a lo anterior, Ariza y Romero (2009a), al referirse a JClic, lo definen como:

JClic es un entorno para la creación, realización y evaluación de Actividades educativas multimedia, desarrolladas en la plataforma Java. Es una aplicación de software libre basada en estándares abiertos que funciona en diversos entornos operativos: Linux, Mac OS X, Windows y Solaris (pág. 45).

El entorno para la creación JClic, está formado por cuatro aplicaciones básicas a través de las cuales se pueden crear, diseñar, editar, modificar, y elaborar actividades multimedia variadas, y las mismas se pueden compartir en la red, estas son:

- JClic applet: El “applet” permite al usuario organizar las actividades JClic en una determinada página web.

- JClic player: Es un programa independiente que al ser instalado permite al usuario crear una serie de actividades multimedia a partir del disco duro de la computadora sin necesidad de tener conexión a internet.

- JClic author: Es una herramienta de autor a través del cual el usuario puede crear, editar, organizar, modificar y publicar las actividades que realice de manera sencilla.

- JClic reports: Es un módulo de recolección de datos que permite la generación de informes sobre los resultados obtenidos en las actividades ejecutadas por los estudiantes.

JClic, es una herramienta muy interesante que permite de acuerdo con Tárraga (2012a): realizar siete (7) tipos básicos de actividades través de la herramienta de Author, y algunas de ellas presentan variantes que permiten la creación de diecisiete (17) posibilidades dentro de las actividades (pág. 124); esto beneficia el proceso pedagógico ya que proporciona variedad en la resolución de actividades con diferentes procedimientos a seguir por parte del 
educando, y al docente le permite variar de acuerdo al contenido y los objetivos.

Siguiendo los aportes del autor citado anteriormente, las actividades que se pueden realizar con el programa son las siguientes: Actividades de asociación simple y compleja, Juegos de memoria, actividades de identificación, puzzles, actividades de texto: completar texto, rellenar agujeros, identificar elementos y ordenar, actividades de respuesta escrita, sopas de letras, crucigramas, y también actividades de aritmética. Como se puede observar, la variedad en las actividades evidentemente coadyuva al enriquecimiento del aprendizaje multidireccional.

Cabe destacar, como señalan Ariza y Romero (2009b), que la idea de implementar JClic: "no es que el alumno diseñe el software sino que lo utilice" (pág. 45); para lograr la construcción de aprendizajes significativos.

\subsubsection{Las principales características de este programa son:}

- Sencillez del entorno Grafico, amigable e intuitivo.

- Fácil manipulación y descarga gratuita.

- Fácil Accesibilidad.

- Compatibilidad con diferentes sistemas operativos (Windows, Mac, Linux) y navegadores variados (Chrome, Opera, Internet Explorer, Mozilla Firefox...).

- Trabajo sin Conexión a Internet.

- Trabajo colaborativo y facilidad para la comunicación síncrona y asíncrona entre alumnos y profesores.

- Las actividades están empaquetadas en proyectos, y contienen la descripción de las actividades, contenidos, y datos del autor e institución. 


\subsection{Bondades e inconvenientes de JClic como herramienta didáctica en la educación primaria}

Sin duda alguna, JClic representa una alternativa bastante llamativa que podría coadyuvar al mejoramiento de las practicas pedagógicas en la educación primaria, por tal razón es necesario resaltar las bondades que ofrece dicho software como herramienta de gran utilidad para el proceso de enseñanza aprendizaje, con el fin de animar a los docentes a explorar las actividades que ofrece y aplicarlas en su campo de trabajo.

A este respecto, señala Santamarina y Fuentes (2017b): considera que la aplicación JClic es la más idónea para ser utilizada por el maestro, por ser de fácil manipulación y representa una alternativa muy llamativa e interesante para trabajar en el aula (pág. 301). A continuación, se muestran algunas de las Bondades, que se obtienen mediante la aplicación de JClic en el aula de clases de Educación Primaria, tanto para maestros como el beneficio que obtienen los estudiantes:

1. El software JClic permite ajustar los niveles de dificultad a los usuarios de acuerdo a un grado específico. El estudiante trabaja de forma autónoma con relación al docente, quien actúa como un facilitador.

2. Las actividades son muy interactivas y poco complicadas, se pueden adaptar a todo tipo de contenidos.

3. Proporciona al usuario, la estructura básica de las actividades a desarrollar, dando oportunidad de que las mismas sean personalizadas por el mismo.

4. Ofrece opciones de retroalimentación al éxito-fracaso, esto incentiva al estudiante a resolver las actividades, o en su defecto a reorientar sus respuestas.

5. Consta de cronometro y contador de intentos, que sirven para evaluar a los estudiantes, si comprenden o no una actividad o contenido.

6. Existe una página web que ofrece información y tutoriales para 
Instituto Internacional de Investigación y Desarrollo Tecnológico Educativo INDTEC, C.A.

DOI: https://doi.org/10.29394/Scientific.issn.2542-2987.2020.5.16.16.305-318

OAI-PMH: http://www.indteca.com/ojs/index.php/Revista_Scientific/oai

Ensayo Original / Original Essay

aprender a manejar el programa, a la vez una comunidad donde se pueden intercambiar ideas y productos.

7. Favorece todos los estilos de aprendizaje, al incluir texto, imágenes, video, sonidos, escritura, etc., donde cada alumno puede trabajar a su ritmo.

8. La elaboración de actividades en JClic requieren de poca inversión de tiempo.

9. No requieren de gran conocimiento informático para utilizar el software.

10.Por último, debo señalar que este software JClic como herramienta en la escuela, ofrece innovación al proceso educativo y motivación en los estudiantes, haciendo énfasis, tal como señalan Santamarina y Fuentes (2017c): que "la motivación, o más bien la falta de ella, por parte de los estudiantes es uno de los principales obstáculos con los que el profesorado se encuentra a menudo en las aulas, y que puede desembocar en fracaso escolar" (pág. 296); y esta herramienta entre otras cosas permite salir de la cotidianidad, y romper con la apatía, y motivar a los educandos que recibirán una educación amena y dinámica.

Por otra parte, como inconveniente, puedo mencionar que esta herramienta al ser utilizada con demasiada regularidad, en un grupo de estudiantes enfocándose en alguna actividad particular, podría llegar a aburrirlos, y le restaría el poder innovador y motivador del programa. Por lo que es recomendable, combinar diversas estrategias y alternar en el uso de varias actividades, manteniendo el estímulo en los educandos.

\subsection{JClic Author y Rol del docente}

JClic Author, es el programa central de JClic, esta herramienta permite a los usuarios, la creación, y edición de materiales educativos multimedia de 


\section{Ensayo Original / Original Essay}

manera fácil y rápida.

En este sentido, destaca Peirats y San Martín (2011), citados por Tárraga (2012b), que:

Este funcionamiento convierte a los programas de autor en una herramienta interesante ya que permite al profesorado elaborar sus propios materiales educativos en soporte digital sin necesidad de tener elevados conocimientos informáticos y realizando un esfuerzo razonable y proporcional a los resultados obtenidos (pág. 123).

De lo antes expuesto, se deduce que la fuerza y empeño que le agregue el docente a la creación de dichos materiales, estará reflejado en la calidad de los mismos al momento de su implementación en el aula, a mayor esfuerzo, mayor será el beneficio que se obtenga a fin de alcanzar el logro de los objetivos propuestos.

Resulta por tanto, de gran importancia que todos los maestros se familiaricen con la herramienta de Author, objeto de esta propuesta pedagógica a fin de incluirla como una alternativa viable que favorezca su papel como facilitador en el aula, a la vez que fomente espacios interactivos de aprendizaje basados en el uso de software limitando así, el uso innecesario de papel, u otros gastos económicos, sacando el máximo provecho que el uso de esta herramienta puede aportar.

Ahora bien, de acuerdo con lo expuesto por Viñals y Cuenca (2016b): señalan como actividades básicas que todo docente debe cumplir y "detectar lo realmente importante, guiar los procesos de búsqueda, analizar la información encontrada, seleccionar la que realmente se necesita, interpretar los datos, sintetizar el contenido y difundirlo son algunas de las tantas tareas que el profesor debe guiar" (pág. 110); expreso mi total acuerdo con los autores precitados, y precisamente cada uno de esos elementos son claves para llevar a cabo un proceso constructivo del conocimiento cónsono con el desarrollo y avance tecnológico en el que actualmente está sumergido la 
escuela, por ende, la figura del docente cobra hoy un papel muy importante, ya que las herramientas tecnológicas sea cual sea, por más ventajosa que sea, ella misma por sí sola, carece de valor sin la presencia y buen uso por parte del docente, quien le da el sentido de uso.

Lo hasta ahora desarrollado me permite aseverar lo imperioso de desarrollar procesos formativos en las instituciones de educación primaria que dirijan sus esfuerzos coherentemente a fin de coadyuvar a la formación del docente en materia de innovación y tecnología, específicamente en el uso de software educativos, como el propuesto JClic permitiendo a los profesionales la oportunidad de crecer, innovar, crear, y educar con prácticas transformadoras que se puedan demostrar con hechos.

JClic Author, constituye entonces, una propuesta pedagógica que podría ser el complemento perfecto al rol que debe ser desempeñado por un docente de actualidad, a la vanguardia de las nuevas herramientas tecnológicas.

\section{Reflexiones finales}

Nos encontramos ante un momento crucial de la historia, el sistema educativo exige innovación, y el llamado tanto de la escuela, como del docente es a innovar, a renovar y actualizarse. La tecnología por sí sola no va a desarrollar las potencialidades de los educandos, ni a realizar las transformaciones en el sistema educativo, depende entonces del uso que le den los docentes desde el punto de vista pedagógico para alcanzar los fines de la educación, es decir formar para vivir en sociedad, en una sociedad marcada por la globalización, y desarrollo tecnológico mediante el uso de metodologías actuales, innovadoras, que motiven a los estudiantes y faciliten la construcción del conocimiento, de forma dinámica e interactiva.

Esta herramienta, JClic, surge como una alternativa bastante llamativa para ser desarrollada en la educación primaria ya que aporta grandes 
beneficios al proceso de enseñanza tanto para los estudiantes como los docentes, quienes al hacer uso de ella, podrán en gran medida salir de lo rutinario, para transformar su praxis pedagógica con materiales multimedia elaborados por ellos mismos, atendiendo las particularidades de un grupo de estudiantes abordando diferentes temáticas o proyectos de aprendizaje en poco tiempo y de manera sencilla.

Con JClic, podrá elaborar actividades básicas como son: las actividades de asociación, de exploración, identificación e información, juegos de memoria, puzles o rompecabezas, actividades de respuesta escrita, actividades de texto, sopas de letras y los crucigramas, algunas de ellas tienen variantes, en cuanto a la presentación de las actividades, lo que convierte a JClic en una herramienta sumamente valiosa y muy interesante para los docentes en educación primaria, por ofrecer variedad y facilidad de uso. Además de ello, existe una comunidad en línea donde se puede compartir experiencias con la aplicación del programa, diversos tutoriales para la creación de actividades con el uso del programa, dándole aplicabilidad, incluso hasta en el bachillerato.

Para concluir, no se puede educar en el Siglo XXI, como se educaba en el pasado Siglo XX, los estudiantes de este siglo están ansiosos por descubrir el mundo para el cual se le está preparando para vivir, por ende, corresponde al docente la tarea de ayudarlos a construir los conocimientos necesarios que coadyuven a la formación del nuevo republicano y nueva republicana, mediante el uso de todo tipo de herramientas que promuevan tal fin.

\section{Referencias}

Ariza, A., \& Romero, S. (2009a,b). El uso del Jclic como complemento para la enseñanza/aprendizaje de la Educación Física. RETOS. Nuevas Tendencias en Educación Física, Deporte y Recreación, (15), 45-48, eISSN: 1579-1726. Recuperado de: 
http://www.redalyc.org/articulo.oa?id=345732280009

Cacheiro, M. (2011). Recursos educativos TIC de información, colaboración y aprendizaje. Pixel-Bit: Revista de medios y educación, (39), 69-81, e-ISSN: 1133-8482. Recuperado de:

https://dialnet.unirioja.es/servlet/articulo?codigo $=3686204$

Núñez, J. (2018). Software JClic como Método de Enseñanza para la Lectura. Revista Scientific, 3(10), 83-94, e-ISSN: 2542-2987. Recuperado de:

https://doi.org/10.29394/Scientific.issn.2542-2987.2018.3.10.4.83-94

Santamarina, S., \& Fuentes, L. (2017a,b,c). Propuesta didáctica con la aplicación JClic para el desarrollo de la comprensión lectora en educación primaria. Revista de Educación de la Universidad de Granada, 24, 293-305, e-ISSN: 0214-0489. Recuperado de:

http://reugra.es/index.php/reugra/article/view/103

Tárraga, R. (2012). JClic y Edilim: programas de autor para el diseño de actividades educativas en soporte digital para Educación Infantil y Primaria. @tic. Revista d'innovació educativa, (9), 123-126, e-ISSN: 1989-3477. Recuperado de:

https://ojs.uv.es/index.php/attic/article/view/1617/1474

Viñals, A., \& Cuenca, J. (2016a,b). El rol del docente en la era digital. Revista interuniversitaria de formación del profesorado, (86), 103-114, ISSN: 0213-8646, e-ISSN: 2530-3791. Recuperado de: https://dialnet.unirioja.es/servlet/articulo?codigo $=5670199$ 


\section{Jerardin del Carmen Ríos Anciani}

e-mail: jerardinrios@gmail.com

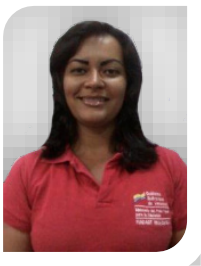

Nacida en Cabimas estado Zulia, Venezuela, el 4 de febrero del año 1985. Obtuve el título de Bachiller en Ciencias en la Unidad Educativa José Isidro Silva; realicé estudios de pregrado en Universidad Nacional Experimental "Rafael María Baralt" (UNERMB), obteniendo el título de Licenciada en Educación Integral en el año 2007; donde también obtuve el título de Magister Scientiarum en Administración de la Educación en el año 2015; graduada como T.S.U. en Informática de la Misión Sucre en la Universidad Bolivariana de Venezuela (UBV, 2019); Desempeño funciones como Docente de Recursos para el Aprendizaje desde el año 2008 hasta la actualidad.

El contenido de este manuscrito se difunde bajo una Licencia de Creative Commons ReconocimientoNoComercial-Compartirlgual 4.0 Internacional 